\title{
Vague Weighted Decision-Making Method and Its Application in Sugarcane Breeding
}

\author{
Hongxu Wang ${ }^{1}$, FuJin Zhang ${ }^{1}$, and Yunsheng $\mathrm{Xu}^{2}$ \\ ${ }^{1}$ College of Science and Engineering, \\ ${ }^{2}$ College of Electronic Information Engineering, Qiongzhou University, \\ Sanya Hainan 572022, China \\ zfj56801@163.com, whx16233@yahoo.cn
}

\begin{abstract}
Similarity measure formula between Vague set $A$ and $B$ is put forward:

$M_{m}(A, B)=\sum_{i=1}^{n}\left[1-\frac{\left|u_{a_{i}}^{(m)}-u_{b_{i}}^{(m)}\right|+\left|v_{a_{i}}^{(m)}-v_{b_{i}}^{(m)}\right|}{2}\right] \cdot\left[1-\frac{\left|t_{a_{i}}^{(m)}-t_{b_{i}}^{(m)}\right|+\left|f_{a_{i}}^{(m)}-f_{b_{i}}^{(m)}\right|}{2}\right]$

Vague weighted decision-making method is the pattern recognition method of the Vague set. It is a weighted decision method applied to sugar cane. Vague strains preferred specific methods and steps are discussed, the preferred method is applied to sugar cane lines. The case shows that these formulas and methods are practical, and comparison with other methods, this one has greater advantages.
\end{abstract}

Keywords: Vague weighted decision-making method, data into formulas, similarity measure formula, sugar cane, Department of preferred varieties.

\section{Introduction}

In 2001, Hainan Sugarcane Breeding Farm bred identified as $B C_{1}$ hybrid strains of Erianthus real sugar cane for the first time. Fuzzy comprehensive evaluation method in Reference [1] studied and analyzed two strains of sugar cane major economic traits of Erianthus hybrids $B C_{1} 17$ to have determined it is the fine lines. Fuzzy comprehensive evaluation method, mainly relying on fuzzy matrix, has a great quantity of calculation. Vague set theory is an extension and development of fuzzy set theory, so in this paper we use vague set theory to study the same problem, with a view to agricultural research issues such as these, given the simplicity of the new research methods, and its application in the agricultural field is of a great potential area. And the formula (1), (2), (3), (4) and (5) put forward in this paper provide the technical support for it.

\section{Basics}

Definition 1 [2] Suppose $\mathrm{C}$ be a collection, $\forall c \in C, \mathrm{G}$ on $\mathrm{C}$ Vague set functions with a true membership function $t_{G}$ and a false membership function $f_{G} \cdot t_{G}(c)$ is from $C$ 
evidence to support the recognition of the degree of membership derived lower bound, while the $f_{G}(c)$ is the evidence from the object $C$ derived the lower bound of the negative membership. $t_{G}(c)$ and $f_{G}(c)$ in the range $[0,1]$ a real number and $\mathrm{C}$ the point of linking that is mapped $t_{G}: C \rightarrow[0,1], f_{G}: C \rightarrow[0,1]$, and $t_{G}(c)+f_{G}(c) \leq 1$. Vague set $\mathrm{G}$ can be recorded at the element $\mathrm{c}$ is $G(c)=\left[t_{G}(c), 1-f_{G}(c)\right]$. Vague can also be abbreviated as $c=\left[t_{c}, 1-f_{c}\right]$.

It can be seen from the definition of $c=\left[t_{c}, 1-f_{c}\right] \subseteq[0,1] . \pi_{c}=1-t_{c}-f_{c}$ represents the element of uncertainty on the Vague Set $G$ function. If $C=\left\{c_{1}, c_{2}, \cdots, c_{n}\right\}$ is a discrete universe of discourse, the Vague on the set $\mathrm{G}$ can be written as $G=\sum_{i=1}^{n}\left[t_{G}\left(c_{i}\right), 1-f_{G}\left(c_{i}\right)\right] / c_{i}$, which can also be abbreviated as $G=\sum_{i=1}^{n}\left[t_{c_{i}}, 1-f_{c_{i}}\right] / c_{i}$.

\section{Vague of the Original Data}

When we solve practical problems with Vague set theory, we must consider the practical problems of the original data to be blurred to create Vague environment, it will not be used to Vague set management Here is one the formula to turn the singlevalue data into Vague data, which will be discussed later on.

Definition 1. Suppose trait evaluation index set is $C=\left\{c_{1}, c_{2}, \cdots, c_{n}\right\}, \mathrm{C}$ is on the set of $G_{i}(i=1,2, \cdots, m) ; \operatorname{target}\left(c_{j}(j=1,2, \cdots, n)\right)$ data for the $c_{i j}(\geq 0)$. If the single-value data $c_{i j}(\geq 0)$ into the data Vague $G_{i}\left(c_{j}\right)=c_{i j}=\left[t_{i j}, 1-f_{i j}\right] \quad$, and $G_{i}\left(c_{j}\right)=c_{i j}=\left[t_{i j}, 1-f_{i j}\right]$ Vague can meet criteria and output criteria, then the singlevalue data into the conversion formula Vague data are called output-oriented transformation formula. If the single-value data $c_{i j}(\geq 0)$ into the data $G_{i}\left(c_{j}\right)=c_{i j}=\left[t_{i j}, 1-f_{i j}\right]$ Vague can mee Vague standards and the investment criteria, then the single-value data into the conversion formula Vague data type conversion formula is known as input. Here:

a. Vague criteria $0 \leq t_{i j} \leq 1-f_{i j} \leq 1$;

b. Output guidelines When $c_{x j}>c_{y j} \geq 0$, rather than negative data $c_{x j}$ and $c_{y j}$ single value, respectively, turning into the Vague data $G_{x}\left(c_{j}\right)=c_{x j}=\left[t_{x j}, 1-f_{x j}\right]$ and $G_{y}\left(c_{j}\right)=c_{y j}=\left[t_{y j}, 1-f_{y j}\right]$, and satisfies: $t_{x j} \geq t_{y j}, 1-f_{x j} \geq 1-f_{y j}$.

c. When $c_{x j}>c_{y j} \geq 0$, rather than negative data $c_{x j}$ and $c_{y j}$ single value, respectively, turning into the Vague data $G_{x}\left(c_{j}\right)=c_{x j}=\left[t_{x j}, 1-f_{x j}\right]$ and $G_{y}\left(c_{j}\right)=c_{y j}=\left[t_{y j}, 1-f_{y j}\right]$, and satisfies: $t_{x j} \geq t_{y j}, 1-f_{x j} \leq 1-f_{y j}$. 
Theorem 1. If the mind $c_{j \max }=\max \left\{c_{1 j}, c_{2 j}, \cdots, c_{m j}\right\}, c_{j \min }=\min \left\{c_{1 j}, c_{2 j}, \cdots, c_{m j}\right\}$, $(i=1,2, \cdots, m ; j=1,2, \cdots, n)$, then:

$$
G_{i}\left(c_{j}\right)=c_{i j}=\left[\frac{c_{i j}}{c_{j \max }},\left(\frac{c_{i j}}{c_{j \max }}\right)^{\frac{1}{3}}\right]
$$

$c_{i j}(\geq 0)$ expressed by the single-value data into the formula is the output Vague data type conversion formula.

$$
G_{i}\left(c_{j}\right)=c_{i j}=\left[1-\left(\frac{c_{i j}}{c_{j \max }}\right)^{\frac{1}{3}}, 1-\frac{c_{i j}}{c_{j \max }}\right]
$$

$c_{i j}(\geq 0)$ expressed by the single-value data into the formula is the output Vague data type conversion formula.

\section{Similarity Measure between Vague Values}

In order to offer the similarity measure between the Vague values, we should recall the data mining Vague value method in Reference [4] first: Vague value $c=\left[t_{c}, 1-f_{c}\right]$ for the definition of $t_{c}^{(0)}=t_{c}, f_{c}^{(0)}=f_{c}, \pi_{c}^{(0)}=\pi_{c}$ :

$$
\begin{aligned}
& t_{c}^{(m)}=t_{c} \cdot\left(1+\pi_{c}+\pi_{c}^{2}+\cdots+\pi_{c}^{m}\right), \\
& f_{c}^{(m)}=f_{c} \cdot\left(1+\pi_{c}+\pi_{c}^{2}+\cdots \pi_{c}^{m}\right), \pi_{c}^{(m)}=\pi_{c}^{m+1} ; \\
& u_{c}^{(m)}=t_{c}^{(m)}-f_{c}^{(m)}, v_{c}^{(m)}=t_{c}^{(m)}+f_{c}^{(m)},(m=0,1,2, \cdots) .
\end{aligned}
$$

The data mining of Vague value, we can construct a similarity measure between Vague value formula.

The so-called constructing Vague environment is turning the raw data into Vague data. This step is a prerequisite for the application of Vague sets. Reference [3] proposed the single-value data into the data definition Vague, here again we mention the assessment of new wheat varieties applying to single-value data into a formula Vague data.

Definition 2. There are two Vague values $a=\left[t_{a}, 1-f_{a}\right]$ and $b=\left[t_{b}, 1-f_{b}\right]$ called the similarity measure between Vague value $A$ and Vague value $B: M(a, b)$, if $M(a, b)$ meets the following criteria:

a. 0 -1 criteria $0 \leq M(a, b) \leq 1$;

b. Symmetric norms $M(a, b)=M(b, a)$;

c. reflexive criteria $M(a, a)=1$;

d. the minimum criteria If when $a=[1,1], b=[0,0]$, or when $a=[0,0], b=[1,1]$, there is $M(a, b)=0$. 
Theorem 2. The following formula is Vague value similarity measures between $a=\left[t_{a}, 1-f_{a}\right]$ and $b=\left[t_{b}, 1-f_{b}\right](m=0,1,2, \cdots)$ :

$$
M_{m}(a, b)=\left[1-\frac{\left|u_{a}^{(m)}-u_{b}^{(m)}\right|+\left|v_{a}^{(m)}-v_{b}^{(m)}\right|}{2}\right] \cdot\left[1-\frac{\left|t_{a}^{(m)}-t_{b}^{(m)}\right|+\left|f_{a}^{(m)}-f_{b}^{(m)}\right|}{2}\right] .
$$

Theorem 3. Let the domain of $C=\left\{c_{1}, c_{2}, \cdots, c_{n}\right\}, \mathrm{C}$ on Vague set $A=\sum_{i=1}^{n}\left[t_{A}\left(c_{i}\right), 1-f_{A}\left(c_{i}\right)\right] / c_{i}$ and $B=\sum_{i=1}^{n}\left[t_{B}\left(c_{i}\right), 1-f_{B}\left(c_{i}\right)\right] / c_{i}$.

They were abbreviated as $A=\sum_{i=1}^{n}\left[t_{a i}, 1-f_{a i}\right] / c_{i}$ and $B=\sum_{i=1}^{n}\left[t_{a i}, 1-f_{a i}\right] / c_{i}$.

Vague set, the following formula is the similarity measure between $\mathrm{a}$ and $\mathrm{b}$ measure $(m=0,1,2, \cdots)$.

$$
M_{m}(A, B)=\sum_{i=1}^{n}\left[1-\frac{\left|u_{a_{i}}^{(m)}-u_{b_{i}}^{(m)}\right|+\left|v_{a_{i}}^{(m)}-v_{b_{i}}^{(m)}\right|}{2}\right] \cdot\left[1-\frac{\left|t_{a_{i}}^{(m)}-t_{b_{i}}^{(m)}\right|+\left|f_{a_{i}}^{(m)}-f_{b_{i}}^{(m)}\right|}{2}\right] .
$$

Note. Vague sets $A$ and $B$ the similarity measure between the value of $M(A, B)$ is used to indicate the similarity between Vague sets $A$ and $B$. The higher the value of $M(A, B)$ is, the more similar of Vague sets $A$ and $B$ are; in particular to obtain the maximum value 1 between $M(A, B)$, then $A$ and $B$, of Vague sets are the most similar. The lower the value of $M(A, B)$, the more dissimilar between Vague sets $A$ and $B$. In particular to obtain the minimum value 0 , then $A$ and $B$, of Vague sets are the least similar.

Theorem 4. If, in the condition of Theorem 3, and note the weighted number of elements in $\omega_{i} \in[0,1]$, and satisfying $\sum_{i=1}^{n} \omega_{i}=1$, then the following formula is the weighted similarity measure between Vague set $Q$ and $P(m=0,1,2, \ldots)$ :

$$
W M_{m}(A, B)=\sum_{i=1}^{n} \omega_{i}\left[1-\frac{\left|u_{a_{i}}^{(m)}-u_{b_{i}}^{(m)}\right|+\left|v_{a_{i}}^{(m)}-v_{b_{i}}^{(m)}\right|}{2}\right] \cdot\left[1-\frac{\left|t_{a_{i}}^{(m)}-t_{b_{i}}^{(m)}\right|+\left|f_{a_{i}}^{(m)}-f_{b_{i}}^{(m)}\right|}{2}\right] .
$$

\section{Vague Program Optimization Methods}

The weighted decision-making method of Vague sets in Reference [6] is constructed into integrated decision-making rules, the general steps of its application are: $\Phi$. Trait evaluation index selection set; $\not$. Extracting to be sorted (or to be preferred) strains programs; 3 . Establishing the most reasonable set of lines program plan; $\theta$. Vague of the original data to get the strains programs Vague sets; 5 . Determining the traits evaluation index weights; 6 Calculating the weighted similarity measure between 
lines to be sorted (or to be preferred) Vague sets and the best lines program; $\not$. the conclusion based on the size of the weighted similarity measure available:

Conclusion 1 the one with greatest value-weighted similarity measure of the program is the best strains; or conclusion 2 Being sorted in accordance with the size of similar sorting program strains. Vague weighted decision-making method is a pattern recognition method.

\section{Case of Its Application}

Re-examine the issue under discussion in Reference [1]with Vague weighted decision-making method.

\subsection{Screening Trait Evaluation Index Set}

Targets set for the judge to determine the characteristics $C=\left\{c_{1}, c_{2}, c_{3}, c_{4}, c_{5}\right\}$, in which $\mathrm{c}_{1}$ : Brix $(\%) ; \mathrm{c}_{2}$ height $(\mathrm{cm}) ; \mathrm{c}_{3}$ : stem diameter $(\mathrm{cm}) ; \mathrm{c}_{4}$ : effective number of stems (of / bundle);: $c_{5}$ empty heart of Po.

\subsection{Extracting the Program to Be Sorted Strains of Sugarcane}

Take care of (then called Guangdong Province, now Hainan Province) sugarcane germplasm nursery of Erianthus a1 strains and hybrids a217 a program composed of strains of sugarcane to be sorted.

Among them, the program $A_{1}$ : strain called YCE01-33; $A_{2}:$ YCE01-34; $A_{3}$ : YCE01-35; $A_{4}$ : YCE01-36; $A_{5}$ : YCE01-37; $A_{6}$ : YCE01-38; $A_{7}:$ YCE01-39; $A_{8}$ : YCE01-40; A : YCE01-41; $A_{10}$ : YCE01-42; $A_{11}:$ YCE01-43;A 12 :YCE01-44; $A_{13}$ : YCE01-45; A 14 : YCE01-46; A 15 : YCE01-47; A 16 : YCE01-48; A 17 YCE01-49.

Table 1. Indicators of economic traits of the model of the original data

\begin{tabular}{cccccc}
\hline program & \multicolumn{5}{c}{ Indicators of economic traits of the model of the original data } \\
\hline & $c_{1}$ & $c_{2}$ & $c_{3}$ & $c_{4}$ & $c_{5}$ \\
\hline$A_{1}$ & 19.1 & 273 & 2.0 & 11 & 1 \\
\hline$A_{2}$ & 19.1 & 276 & 2.3 & 1 & 2 \\
\hline$A_{3}$ & 23.4 & 249 & 2.4 & 4 & 1 \\
\hline$A_{4}$ & 18.6 & 159 & 1.8 & 6 & 2 \\
\hline$A_{5}$ & 18.6 & 242 & 2.1 & 10 & 1 \\
\hline$A_{6}$ & 16.4 & 127 & 2.6 & 3 & 1 \\
\hline$A_{7}$ & 21.6 & 175 & 1.6 & 10 & 3 \\
\hline$A_{8}$ & 19.5 & 169 & 2.5 & 2 & 1 \\
\hline$A_{9}$ & 13.7 & 165 & 2.0 & 4 & 1 \\
\hline$A_{10}$ & 18.2 & 174 & 1.9 & 2 & 1 \\
\hline
\end{tabular}


Table 2. Indicators of economic traits of the model data Vague

\begin{tabular}{cccccc}
\hline program & \multicolumn{5}{c}{ Indicators of economic traits of the model data Vague } \\
\hline & $c_{1}$ & $c_{2}$ & $c_{3}$ & $c_{4}$ & $c_{5}$ \\
\hline$A_{1}$ & {$[0.816,0.934$} & {$[0.989,0.996]$} & {$[0.989,0.996]$} & {$[1.000,1.000]$} & {$[0.307,0.667]$} \\
\hline$A_{2}$ & {$[0.816,0.934]$} & {$[1.000,1.000]$} & {$[0.885,0.960]$} & {$[0.091,0.450]$} & {$[0.126,0.333]$} \\
\hline$\ldots$ & $\ldots$ & $\ldots$ & $\ldots$ & $\ldots$ & $\ldots$ \\
\hline$A_{17}$ & {$[0.808,0.931]$} & {$[0.783,0.922]$} & {$[0.808,0.931]$} & {$[0.636,0.860]$} & {$[0.000,0.000]$} \\
\hline$B$ & {$[1.000,1.000]$} & {$[1.000,1.000]$} & {$[1.000,1.000]$} & {$[1.000,1.000]$} & {$[0.307,0.667]$} \\
\hline
\end{tabular}

\subsection{Establishing the Most Ideal Strains of Sugar Cane Program}

As in the strains of economic traits in the evaluation index, the Brix, plant height (stem diameter and the effective number to the value stems are much good); and empty heart of the small value of Po for the best, so the strain evaluation index, the best economic traits data can be composed of the best strains of sugar cane program, denoted by B. The strains of sugar cane, cane pattern data and the ideal scenario B strains of the original data are listed in Table 1 (from Reference [1]).

\subsection{Vague of the Orginal Data}

In the formula (1) and (2), turn the Table 1 into Table 2.Table 2 shows the various strains of sugarcane strains aaab and best mode B, Vague set of data.

\subsection{Calculating the Similarity Measure between the Program of the Vague Set to Be Sorted and the Most Ideal Program of Vague Set}

Reference [1] gives the index of the weighted factor traits: $c_{1}$ (Brix) of $0.30 ; c_{2}$ (height) of $0.10 ; c_{3}$ (stem diameter) of $0.15 ; c_{4}$ (effective number of stems) is $0.30 ; c_{5}$ (empty Po heart level) is 0.15 ; and take parameters $m=2$, application of the formula (8). Calculate the strain of sugarcane Vague set to be sorted and the best solution a1 a17 strains of sugar cane similarity measure between scenario $B$, the results are as follows:

$$
\begin{aligned}
& W M_{2}\left(A_{1}, B\right)=0.899, W M_{2}\left(A_{2}, B\right)=0.567, W M_{2}\left(A_{3}, B\right)=0.775, \\
& W M_{2}\left(A_{4}, B\right)=0.677, W M_{2}\left(A_{5}, B\right)=0.899, W M_{2}\left(A_{6}, B\right)=0.619, \\
& W M_{2}\left(A_{7}, B\right)=0.761, W M_{2}\left(A_{8}, B\right)=0.645, W M_{2}\left(A_{9}, B\right)=0.602, \\
& W M_{2}\left(A_{10}, B\right)=0.603, W M_{2}\left(A_{11}, B\right)=0.824, W M_{2}\left(A_{12}, B\right)=0.844, \\
& W M_{2}\left(A_{13}, B\right)=0.511, W M_{2}\left(A_{14}, B\right)=0.878, W M_{2}\left(A_{15}, B\right)=0.547, \\
& W M_{2}\left(A_{16}, B\right)=0.620, W M_{2}\left(A_{17}, B\right)=0.700 .
\end{aligned}
$$


The purpose to calculate the similarity measure is: comparing each program to be sort of sugarcane strains $A_{i}(i=1,2, \cdots, 17)$ with the best sugar cane program model $\mathrm{B}$, the first value is the first priority lines, the second number is the second priority strain, ..., the minimum value is ranked in the final lines, so according to the above results, the strains of sugar cane obtained to be sorted are as follows:

A1 and A5 tied 1, A4 No. 3, A12 No. 4, A11 No. 5, A3 No. 6, A7 No. 7,..,A15 No. 17.

The conclusion both in this paper and in Reference [1] are basically the same, but the fuzzy comprehensive evaluation method in Reference [1] requires a lot of matrix calculations with a large quantity of the calculation. This method in this paper is simple and replace fuzzy evaluation method.

\section{Conclusion}

The similarity measure formula between Vague sets discussed in this paper provides more choice of means for fuzzy information processing, but from the application data we can see the two similarity measure conversion formula turning a single value into Vague data sets is the referred method of the two basic options. Vague program for the study of the preferred method also provides a new method to solve the issues related to agriculture, which is an alternative method of fuzzy comprehensive evaluation method.

Acknowledgments. The Hainan Provincial Natural Science Fund Project No.610224; the Hainan Provincial Social development projects for science and technology development fund No.2010SF004 and Sanya City College, 2009 special fund project funding issues (YD09027).

\section{References}

1. Liu, S.-M., Fu, C., Huang, Z.-X.: Fuzzy comprehensive evaluation on the clones of Erianthus arundinaceou. Guangdong Agricultural Science (8), 16-18, 21 (2008)

2. Gau, W.-L., Buehrer, D.J.: Vague Sets. IEEE Transactions on Systems, Man and Cybernetics 23(2), 610-614 (1993)

3. Wang, H.-X.: Definition and transforming formulas from the single valued data to the vague valued data. Computer Engineering and Applications 46(24), 42-44 (2010)

4. Liu, H.-W., Wang, F.-Y.: Transformations and Similarity Measures of Vague Sets. Computer Engineering and Applications 40(32), 79-81, 84 (2004)

5. Wang, H.-X.: Similarity measure between vague sets and their application. Computer Engineering and Applications 46(26), 198-199 (2010)

6. Wang, H.-X.: Synthesis decision rule of vague sets and its application in scheme optimum seeking. Computer Engineering and Applications 46(27), 145-147 (2010) 